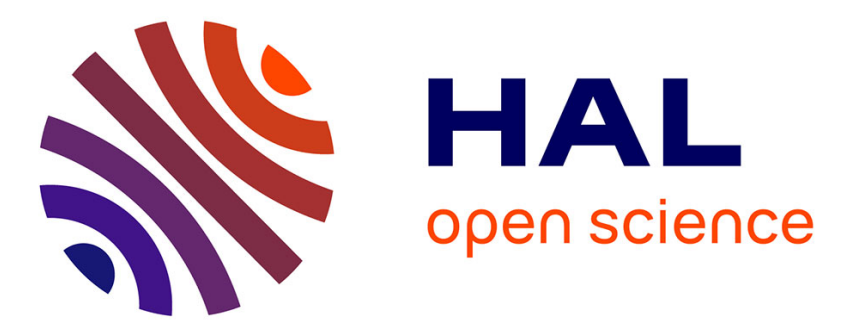

\title{
"Sconfinato è solo il niente" Le Je entre fracture et récognition dans Vista dalla luna de Chandra Livia Candiani
}

\author{
Ilaria Moretti
}

\section{- To cite this version:}

Ilaria Moretti. "Sconfinato è solo il niente" Le Je entre fracture et récognition dans Vista dalla luna de Chandra Livia Candiani. Studia Universitatis Babes Bolyai - Studia Philologia, 2019, LXIV, 4, 2019, p. 271 - 287. halshs-02410987

\section{HAL Id: halshs-02410987 \\ https://shs.hal.science/halshs-02410987}

Submitted on 14 Dec 2019

HAL is a multi-disciplinary open access archive for the deposit and dissemination of scientific research documents, whether they are published or not. The documents may come from teaching and research institutions in France or abroad, or from public or private research centers.
L'archive ouverte pluridisciplinaire HAL, est destinée au dépôt et à la diffusion de documents scientifiques de niveau recherche, publiés ou non, émanant des établissements d'enseignement et de recherche français ou étrangers, des laboratoires publics ou privés. 


\section{«Sconfinato è solo il niente» \\ Le Je entre fracture et récognition dans Vista dalla luna de Chandra Livia Candiani}

\section{ILARIA MORETTI ${ }^{1}$}

\section{Une carte heuristique pour sonder l'abîme}

Au mois d'avril 2019, la poétesse Chandra Livia Candiani revient sur la scène éditoriale italienne grâce à la publication d'un nouveau recueil de poèmes : Vista dalla luna (Salani, 2019)². Le livre, composé de deux parties différentes, affronte la thématique de l'enfance souffrante à travers deux axes complémentaires. Dans la première partie - Vista dalla luna - le lecteur se trouve confronté à une série de poèmes écrits entre le mois de janvier 1999 et le mois d'avril 2000. La protagoniste est une jeune fille nommée $I o$ : « Je ». Il s'agit d'un sujet privé de souvenirs. Elle vient de la lune, comme il lui rappelle sans cesse sa mère. Elle n'est pas de cette terre, elle n'est pas une jeune fille comme les autres. Elle n'a pas de mémoire ni de points de repère qui lui permettent de déchiffrer l'univers des adultes. C'est sa mère - figure de la folie, mais, avant tout, figure de l'absence - qui la condamne à cette errance perpétuelle. C'est ainsi qu' $I o$ - ce $J e$ en quête de réponses - cherche à construire une sorte de carte personnelle pour s'orienter, pour comprendre les autres et se définir. Il s'agit d'un plan mental, d'une carte heuristique qui fait des objets quotidiens, des mots, des animaux, des plantes, les points de contact nécessaires pour survivre « après la bombe ${ }^{3}$ ». Candiani nous apprend la possibilité de se structurer après une catastrophe représentée, pour elle, par une enfance ravagée par les absences, les non-dits, et un manque de cure qui finit pour devenir un manque d'amour.

Adoptant une perspective complémentaire, la deuxième partie du volume se structure autour de deux figures : un petit garçon - « il bambino » - et une petite fille, « la bambina ». Le poème La porte, écrit entre le mois de décembre 2005 et le mois de janvier 2006, avait déjà été publié dans La biblioteca di Vivarium en 2006 mais il trouve, dans ce contexte, un nouveau souffle.

\footnotetext{
${ }^{1}$ Doctorante en littérature italienne contemporaine (ED 487 : Philosophie, Histoire, Création, Représentation) et LECTRICE d'italien à l'Université Jean Moulin Lyon 3 (IHRIM - UMR 5317), elle a été ATER dans la même université pendant la période 2017-2019. Sa thèse porte sur l'œuvre littéraire d'Elena Ferrante analysée par le biais des personnages féminins à la lumière de la métabiographie et de la philosophie de la narration. Diplômée à l'Université de Bergame en Cultures Modernes et Comparées elle est spécialiste de littérature italienne contemporaine avec un axe d'étude centré sur l'écriture féminine et l'auto-narration. Elle est chroniqueuse littéraire pour Charta Sporca (Université de Trieste) et La Clé des langues et elle a collaboré avec Il fascino degli intellettuali, Doppiozero, Soft Revolution, Il lavoro culturale, Cronache letterarie, Critica letteraria. Elle a créé et dirige actuellement l'Atelier de théâtre en Italien de l'Université Jean Moulin Lyon 3.

${ }^{2}$ C. Livia CANDiAni, Vista dalla luna, Milano, Salani Editore, 2019.

${ }^{3}$ Voir à ce propos l'interview réalisé par Italian Reloaded le 2015 à San Vito di Cadore (BL), Chandra - La precisione della poesia : https://www.youtube.com/watch? $=$ WzGpck52vXE\&t=1394s.
} 
Dans sa brévité, dans sa mise en valeur d'une parole essentielle qui procède avec un rythme saccadé, ce poemetto constitue le naturel prolongement thématique du recueil Vista dalla luna.

L'enfance est à nouveau un lieu obscur, traversé d'incompréhension et de violence. Le vide affectif est incarné, cette fois-ci, par la figure d'un assassin qui n'est pas un étranger, un inconnu venu de loin, mais un semblable : un proche. Il se cache derrière le mur, il porte dans sa configuration énigmatique les traces de l'incertitude et de l'angoisse. La porte, qui sépare l'assassin de petits protagonistes, n'est qu'une barrière fragile ; une séparation entre la candeur et l'horreur. Elle est le palliatif qui donne l'illusion d'un abri qui, malgré tout, se révèle inutile. Car, dans ces courts poèmes, l'ogre - colonisateur d'une terre protégée - habite la même maison de ses enfants. L'espace privatif devient, ainsi, un terrain de lutte ; un lieu d'insécurité où les murs s'écroulent, où le terrain glisse sous les pieds. La porte du titre n'est donc pas celle qui donne sur l'extérieur, qui sépare le public du privé. Mais celle qui divise une chambre de l'autre. L'assassin ne peut être qu'un membre de la famille ; c'est quelqu'un qui devrait protéger ses petits et qui finit, par un dramatique jeu d'opposition, par dévoiler sa grimace de bourreau.

Avec son volume Vista dalla luna Candiani revient, donc, sur l'une de ses thématiques fondatrices : l'enfance et ses mystères, ses angoisses, ses multiples possibilités. C'est la même enfance déjà narrée dans le recueil La bambina pugile (Einaudi, 2014). Nous faisons référence à une enfance qui cherche à structurer l'individu, qui le forge malgré la douleur, les blessures, au-delà d'une souffrance qui étouffe et qui semble condamner le sujet au spectre de l'incommunicabilité. La poétesse, dans La bambina pugile, avait déjà suggéré une réponse possible pour contraster l'abîme du sens, le marécage de l'impossibilité. Il faut savoir suivre des plans, des authentiques «mappe » capables de nous guider vers une forme de récognition qui est à la fois identitaire et intellectuelle. La carte heuristique, construite grâce à la poésie, traduit la volonté de s'imposer une discipline. Car, pour Candiani, la seule manière pour faire face à l'abandon est de commencer par l'écoute, par l'ouverture à l'autre, par l'observation d'un monde qui se forge en dépit de sa fracture. Les deux poèmes, Mappa per l'ascolto et Mappa per pregare ${ }^{4}$, publiés dans La bambina pugile, montrent la nécessité de repenser le monde à partir d'une perspective mineure : liminaire. Il faut savoir lire dans le vol des hirondelles la marque d'une émancipation subjective. Il faut apprendre la discipline secrète qui nous permet de transformer notre propre main en une coquille : prothèse auditive, outil de perception.

\footnotetext{
${ }^{4}$ C. Livia CANDiani, La bambina pugile, Torino, Einaudi, 2014, p. 14-19.
} 
Le chemin indiqué par Candiani s'ouvre sur un apprentissage au silence : le sujet, fondu à la nature, apprivoise ce bruit sourd et feutré qui se produit dans l'absence. Il s'agit de ce même vide qui emprisonne non seulement l'enfant, mais tout individu. La poétesse met l'accent sur un néant qui détruit et qui, soudainement, se fait révélateur de sens. Savoir l'observer - mieux : l'écouter - permet au sujet d'en saisir la complexité. C'est ainsi que cette cavité obscure se révèle être porteuse d'autres voix : celles des mots. Le vide laisse ressurgir la richesse inespérée d'une série de « parole in boccio ${ }^{5}$ » capables de fleurir après le dégel. Elles percent la solitude d'une longue nuit affective en permettant au sujet de se faire maître de sa propre histoire :« sei tu / che di notte scegli ${ }^{6} \gg$.

Et encore. L'enfance dans Vista dalla luna est, pour reprendre une expression de Chandra

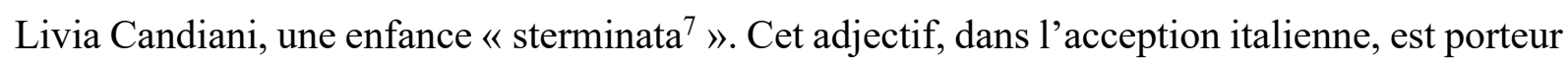
de deux significations distinctes : exterminée et illimitée. L'enfance se configure comme un lieu qui n'a pas de limites, qui n'a pas de temporalité. Parallèlement - dans sa signification la plus sombre - elle représente une saison qui peut être anéantie, détruite par la main d'un autrui sans scrupules ; un inconnu incapable de comprendre. Un homme désireux de punir, blesser, brutaliser.

C'est ainsi que Chandra met en place, dans son nouveau recueil, une tentative de préservation de l'enfance. Enfance interprétée, donc, comme lieu de formation, mais aussi comme terre précaire que les êtres humains - tous - doivent préserver. Sa voix poétique se lève en opposition à la suppression de l'imagination. Elle dénonce la violence quotidienne que les enfants subissent, partout dans le monde. Une violence qui est avant tout physique, où l'enfant est traité (maltraité !) comme un objet: il est observé, touché, usé, abusé, violé ${ }^{8}$. Mais sa critique, son cri à la fois désespéré et chargé d'un espoir concret - engagé -, s'adresse aussi à l'école, le lieu qui enseigne aux enfants « a non sentire ${ }^{9} »$. Comme nous le rappelle Chandra, c'est un cri qui se lève dans la volonté de réveiller les parents. Eux, les adultes, souvent plongés dans leurs smartphones, ne s'aperçoivent pas que les enfants, seuls, regardent dans le vide.

Ces poésies, qui mélangent l'atrocité au sublime du quotidien, nous montrent comment la parole enfantine peut être interprétée comme une sorte de prophétie. Les enfants, pour Candiani, sont ces petits prophètes de la réalité - « profeti della realtà ${ }^{10} »-$ qui apprennent une capacité

\footnotetext{
${ }^{5}$ C. Livia CANDIANI, « Mappa per l'ascolto » La bambina pugile, op. cit., p. 14.

${ }^{6}$ C. LIVIA CANDIANI, « Mappa per pregare $»$, La bambina pugile, op. cit., p. 19.

${ }^{7}$ C. Livia CANDIANI, « Esseri visti dalla luna », Vista dalla luna, op. cit., p. 9.

${ }^{8}$ Ivi.

${ }^{9}$ Ibid., p. 10.

${ }^{10}$ Ivi.
} 
de vision que les adultes semblent avoir perdue. L'enfance - cette même enfance rêvée, saccagée et puis, finalement, retrouvée - ne devient rien d'autre qu'une posture, un lieu d'observation offrant une perspective privilégiée capable de détruire toute barrière temporelle. Chandra s'adresse à ceux qui croient que pour comprendre le monde il faut être capable de « stare nel presente ${ }^{11}$ ». L'enfance est, contrairement aux apparences, un moyen d'habiter ce présent tant demandé ; c'est une manière de l'affronter par le biais d'une optique qui n'impose rien. Elle cherche, plutôt, à comprendre. Le présent de l'enfance est cette capacité à savoir « giocare su tutto ». C'est la joie qui se structurer autour de la douleur : une joie démesurée qui «fa crepare d'invidia gli adulti di professione ${ }^{12}$. Saisir l'enfance comme angle de lecture montre, pour Chandra, le besoin d'assumer son propre destin, sans oublier une qualité fondamentale : celle de pouvoir continuer à danser - légers - contre la fatigue du quotidien. L'enfance est, finalement, une vocation qui se traduit dans un plaisir de connaissance étrange et solitaire.

Car, il ne faut pas oublier que dans ces pages brûlantes - qui alternent, sans cesse, la candeur au dégoût - Candiani raconte d'elle-même. Sa page devient un terrain propice pour mettre en place une métabiographie en poésie. Elle fait de son récit personnel un prétexte pour réfléchir sur son œuvre. Elle utilise la page pour étudier les multiples possibilités d'une parole, d'une langue, qui intervient - là - où le monde semble avoir perdu toute faculté d'expression. Car, en lisant ses poèmes, un rapprochement nous paraît évident. Cette petite fille venue de la lune n'est peut-être rien d'autre que Chandra elle-même. Cette même Chandra qui porte un prénom issu de la tradition indienne signifiant, en effet : lune ${ }^{13}$. Cela signifierait que cette petite fille, appelée - emblématiquement - « Io », incarne son Je lyrique. Un Je de poète qui se fait personnage et qui revendique la place, centrale, assumée par la première personne.

En suivant cette optique, il est important de rappeler que, pour Candiani, il existe une relation mystérieuse entre biographie et création poétique. Elle l'explique dans sa préface au volume en montrant comment c'est l'œuvre, tout compte fait, qui rêve de la vie. C'est elle qui, parfois, la suit : parfois la précède ${ }^{14}$. L'œuvre dicte le parcours d'une existence qui se concrétise grâce à la parole. La poétesse est, alors, une conteuse de mondes, de son monde : elle « indoss[a] la [sua] storia e la port[a] sempre con $[\text { sé}]^{15} \gg$. Comprendre son propre vécu pour en faire matière

\footnotetext{
${ }^{11}$ Ibid., p. 11.

${ }^{12}$ Ivi.

${ }^{13}$ ItAliAn RELOADED, «Luna », Chandra - La precisione della poesia, op. cit.

${ }^{14}$ C. Livia CANDIANI, « Esseri visti dalla luna », op. cit., p. 14.

${ }^{15} \mathrm{Ivi}$.
} 
de poésie, semble être, finalement, l'impératif que Candiani se donne pour pouvoir transformer la souffrance en possibilité.

La littérature est un moyen, peut-être le plus efficace, pour trouver la force de regarder son propre parcours en acceptant les coins d'ombre. Car, finalement, même la douleur la plus aiguë révèle, dans sa férocité, un brin de lumière. Grâce à la narration, toute souffrance peut devenir lueur : occasion de réparation. Elle «buss[a] alla [su]a storia e la fiss[a] negli occhi finché diventa luce ${ }^{16} \gg$.

À partir de ce constat, nous pouvons donc nous demander par quel biais ce Je narratif - cette enfant venue de la lune et appelée « Io » - parvient à la construction de sa propre identité à travers la poésie. Nous faisons référence à une subjectivation qui est, certes, ancrée dans la parole mais qui se fait, aussi, véritable moyen de récognition existentielle. C'est par ce parcours que nous chercherons à monter comment la poésie devient, pour Candiani, un important outil sémantique capable de redonner sens - et donc dignité - à un sujet fracturé. La poésie devient, dans ce contexte, un instrument capable de structurer l'individu - dans notre cas la femme, l'écrivaine, la fille - en dépit de son identité en lambeaux. À travers une analyse métabiographique, nous verrons comment Chandra arrive à mettre en place, dans les poèmes de Vista dalla luna, une sorte de sémantique affective qui se traduit dans la quête d'un ordre existentiel, nécessaire à sa survie. Cet ordre donné par le langage est l'arme que l'enfant protagoniste - « Io »-oppose à la folie dans laquelle elle a grandi. Il s'agit de la folie du monde, mais surtout la folie de ses parents. Candiani, dans ses vers pétris d'âpreté et de splendeur, fait preuve d'une discipline intellectuelle qui lui permet de transformer la fracture congénitale en paradigme de sens, car, finalement : l'identité vient des mots.

\section{Crier la folie des origines : parentalité et orphanité}

Pour comprendre la poésie de Candiani et pour saisir les spécificités du personnage raconté dans Vista dalla luna, il est important de se concentrer sur une donnée biographique que l'auteure n'a jamais cessé de revendiquer tout au long de ses interviews. Chandra est née au sein d'une famille de fous. Elle l'a précisé sans vergogne, sans crainte d'utiliser des mots qui peuvent paraître brutaux. Sa famille ne lui a pas donné la possibilité de s'orienter. Elle n'a pas eu de plans : pas de «mappe » pour comprendre le monde ${ }^{17}$. Même le langage, le rapport aux objets, la capacité de vision, étaient altérés par une éducation au négatif : une éducation née

\footnotetext{
${ }^{16} \mathrm{Ivi}$

${ }^{17}$ Italian Reloaded, « Famiglia », Chandra - La precisione della poesia, op. cit.
} 
sous l'enseigne de l'obscurité. C'est dans ce contexte que Chandra, dès son plus jeune âge, a dû élaborer la barbarie - la violence immotivée - en cherchant à la traduire dans une langue personnelle. Une langue autre - parallèle - à celle de parents. Elle a dû se construire contre la Langue Maternelle - la Langue de la Mère - en édifiant un alphabet personnel capable de signifier les mots-creux laissés en héritage par sa famille. La poésie, pour elle, est la langue de tous ceux qui ne savent pas parler ${ }^{18}$. De tous ceux qui ont perdu les mots ou qui ne les ont jamais possédés. Chandra se structure, à l'instar de son Je poétique, en acceptant l'incompréhension et en faisant de cette matière douloureuse une possibilité de survie. L'horreur a été affrontée à l'instar d'un travail quotidien. Savoir le déchiffrer a été la seule manière pour rétablir un ordre dans le chaos affectif.

Dans Vista dalla luna, « Io », l'enfant protagoniste, se trouve dans une situation analogue. Deux figures menacent son enfance : le père et la mère. Il s'agit d'une enfance où « i grandi si sbranano ${ }^{19} »$, où les phrases entre guillemets coupent le discours en produisant une altération du rythme. Le lecteur se heurte à la parole de Candiani : une parole essentielle qui devient, soudainement, brutale. Son langage alterne la simplicité d'un idiome quotidien, à la violence des menaces, des injures. La sobriété de l'expression enfantine s'oppose à la véhémence d'une langue étrangère : celle des adultes.

Un homme crie : «Puttana vestita di nero », «Ti ammazzo di botte ${ }^{20}$. Ce sont des expressions qui se dirigent, potentiellement, vers une figure maternelle absente, inerme, incapable de réagir. Le père raconté dans ces pages est un « $\operatorname{orco}^{21} »$, un ogre qui revient toujours avec les mêmes menaces. Le Je poétique reste étonné face à une série de phrases qu'il n'arrive à déchiffrer. Ces expressions délimitées par les guillemets se présentent, donc, comme une lacération au sein du discours. Elles brisent, dans leur férocité, la tentative de reconstruction de ce Je qui se bat à l'intérieur d'un univers privé d'amour. Le septième poème commence par : « Ti spezzo in due / te ne do tante da levarti il fiato ${ }^{22}$ ». « Io » ne sait pas comment réagir. Elle ne comprend pas si cette parole brutale, qui marque le rythme de chaque journée, est adressée contre un inconnu ou contre la mère : sa propre mère. Autre possibilité : ces menaces, qui ne donnent pas d'alternatives, sont peut-être adressées à la protagoniste elle-même. Elles visent l'enfant de la lune ; celle qui n'a pas de points de repère sur cette terre. Celle qui est perdue.

\footnotetext{
${ }^{18}$ ITALIAN RELOADED, « La poesia », op. cit.

${ }^{19}$ C. LiVIA CANDIANI, « Vista dalla luna », op. cit., p. 18.

${ }^{20}$ Ivi.

${ }^{21}$ Ibid., p. 19.

${ }^{22}$ Ibid., p. 23.
} 
Mais l'ogre ne se limite pas aux injures. Il est un homme d'action et dans certains poèmes il se lance contre «Io » sans pitié. Il détruit, ainsi, la seule possibilité de relation qu'il pouvait instaurer avec son enfant. Les repas se transforment, donc, en véritables scènes de torture : «l'orco che mi inghiotta / in un boccone e non mi imbocchi / ferendo la forchetta di rossa / rugiada $^{23} »$. Le père peut dévorer et, parallèlement, monter son mépris, son incapacité de cure. La fourchette, utilisée pour nourrir l'enfant, se teint de rouge. C'est le sang qui jaillit à la suite d'une blessure, mais ce rouge intense - si vif - devient, en poésie, léger comme la rosée du matin. Se sauver signifie, pour Candiani, - et, transversalement, pour son Je poétique transposer la barbarie en faisant de l'atrocité un prétexte de création. Si la goutte de sang s'associe à la rosée du matin, alors la bouche blessée n'est rien d'autre qu'une fleur capable de renaître à chaque lever du soleil. Il existe une possibilité de fuite face à l'abjection. Cette ouverture est incarnée par l'imaginaire, par les objets du monde, les plantes, la nature: véritables maîtres capables de diriger le regard, orienter la pensée.

Mais ce père, l'ogre de l'enfance - le barbare - n'est finalement qu'une figure de passage. Il est un être « imprestato ${ }^{24} »-$ un 'emprunté' - le fruit d'un hasard qui ne doit pas conditionner la totalité d'une existence. À l'instar de la mère-aveugle, il est une figure liminaire. Il représente la cassure émotive qui traverse le sujet - l'enfant, dans ce contexte - mais il peut être, aussi, renvoyé à son univers lacunaire. Sa voix de tyran est une voix qui n'arrive pas à étouffer l'intégrité d' « Io ». Sa carence affective revient, aussi, dans la figuration de sa mort. Le cercueil du père est déterré - « La bara / del padre morto è insepolta ${ }^{25} »-$ et il se retrouve dans une chambre funéraire partagée avec d'autres pères, d'autres mères. Ces parents morts sont tous coupables de lacunes relationnelles. Tous affectés par la même incapacité d'amour : " nella camera ardente con altri / padri madri, nessuno arde, solo / le parole di Io dentro il petto ${ }^{26} »$.

C'est ainsi que les mots d'« Io » perdurent contre la fragilité de deux figures parentales si incompréhensibles. Toutefois le père - qui dans le petit poème La porta revient sous les apparences d'un assassin - est celui qui se bat, sans cesse, contre la naissance de ces mêmes mots. L'assassin est celui qui coud la bouche - «cuce la bocca ${ }^{27} »-$ pour détruire le développement du langage. C'est un tueur d'expressions ; un être qui revendique sa volonté de suppression de tout chemin communicatif. Son ennemi est cette parole dangereuse qui garde, dans sa polysémie, un pouvoir thérapeutique. Dans son optique il est nécessaire de couper-

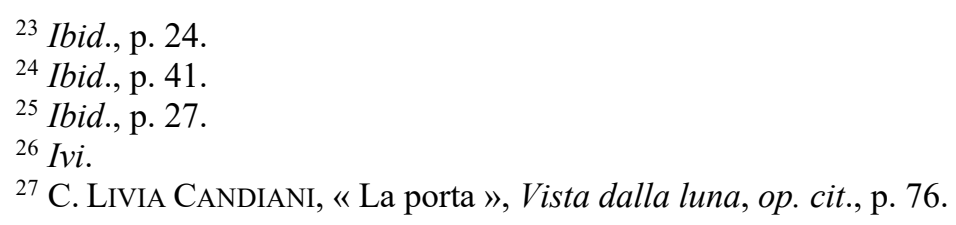


d'interdire, même à travers la force - toute tentative d'échappatoire à son emprise affective. L'assassin se configure, ainsi, comme un père aveugle et sourd qui, face aux demandes d'amour, préfère tourner le regard. Caché derrière la porte, il observe ses victimes. La porte, qui donne le titre au poème, n'est rien d'autre que la métaphore d'un espace à la fois métaphorique et réel. Comme nous l'avons vu, elle est la barrière infranchissable apte à protéger l'esprit de toute invasion. Ou encore, elle est interprétée comme l'élément tangible qui délimite le périmètre entre deux chambres : celle des petits et celle des adultes. Car, dans ce poème, l'assassin porte - sournoisement - le même pyjama de son enfant : « La bambina. / [...] Aveva lo stesso pigiama. / Dell'assassino. / Ma più piccolo ${ }^{28} »$.

Dans ces poèmes, il n'existe pas de zone frontalière entre le bien et le mal. L'univers de Candiani est un lieu de transition et de contamination. Le monde familial se présente comme un tourbillon de fragments affolés. Le besoin d'amour et d'empathie se fusionne au désir de détachement. Dans cette incertitude, la parole semble être la seule possibilité de survivance face au chaos : elle s'élève contre l'incohésion affective et les dérives du déchirement relationnel.

D'une manière parallèle, la figure maternelle assume une place singulière à l'intérieur de ce même univers parental. En effet, elle se configure comme un contre-chant à la brutalité du père. Sa présence ne s'impose pas à travers la violence physique ; pour autant, elle n'est pas moins coupable. La mère, dans Vista dalla luna, se fait allégorie du sentiment d'abandon. Il s'agit d'une mère-ombre : elle incarne le silence qui se fait - pour paraphraser Candiani - chose vivante $^{29}$. Cette figure impalpable ne représente pas une forme d'apaisement. Au contraire : elle oppose un mutisme permanent, inébranlable, aux questionnements de sa fille. La mère se définit à travers deux représentations différentes. D'un côté nous avons la mère-aveugle qui, avec son attitude, se fait porteuse d'une éducation à la perte. Il s'agit d'une éducation au vide qui contraint l'enfant - « Io » - à faire face à un manque de réponses. Elle précipite, ainsi, dans le marécage du non-sens affectif. De l'autre côté, la figure maternelle se fusionne au spectre du père, mieux : de l'assassin. Elle en devient la prolongation; elle s'incarne dans sa férocité déraisonnée, dans son absence de patience, dans son désert intellectuel.

C'est ainsi que le Je poétique de Vista dalla luna se lève contre une matrice incapable de présence. La poésie de Candiani assume, dans ces pages, des contours opaques, alternant la candeur de l'enfance à la précarité de la raison. Le sang, la dépossession, des scènes de violence

\footnotetext{
${ }^{28}$ Ibid., p. 81.

${ }^{29}$ Voir à ce propos le titre d'un récent essai poétique de C. Livia CANDIANI, Il silenzio è cosa viva, Torino, Einaudi, 2018.
} 
coupent le rythme du discours en ouvrant à des perspectives à la fois opposées et complémentaires. Dans cette deuxième partie, la mère-aveugle devient, donc, une mèrecannibale qui finit par englober l'autre. Elle le dévore - elle le supprime - à cause de son manque d'amour.

Concernant le premier paradigme, nous observons comment le silence maternel s'associe à son incapacité de vision. L'aveuglement de la mère revient dans plusieurs poèmes. Elle est entourée par un brouillard sans fond, sans limites : «C'è la nebbia mamma, / tu non la vedi ma Io ti accompagna ${ }^{30} »$. À cause de son égarement sensoriel et émotif, la mère n'arrive pas à entrevoir le profil de sa propre fille. Toutefois ce brouillard, si persistant, si tenace, n'existe que dans son optique de malade, de folle. « Io » est là. Malgré la brume elle observe sa mère. Son regard est lucide : elle perçoit sa perdition, son incapacité de reconnaissance. Elle voit et ce discernement l'oblige à se confronter à cet autrui parental qui devrait la guider - la préserver et qui, à l'inverse, est perdu dans l'obscurité. La mère d'« Io » possède des yeux sombres : inutile de chercher. Dans la profondeur de son iris, il n'y a pas d'existence. Pas de lueur, pas de possibilités. Ses yeux sont vides : «non ti affacciare / agli occhi della mamma ${ }^{31}$ ». En même temps, sa posture reste celle d'une femme qui, en débit de ses carences, choisit, délibérément, de rester dans son désert affectif. Elle ne veut pas voir. Elle tourne souvent le dos à sa fille «[...] la mamma girata / dall'altra parte ${ }^{32} »-$ et refuse, ainsi, de pénétrer dans l'univers d'« Io ». Elle parvient même à l'accuser. Selon son optique, c'est « Io » qui ne veut pas être comprise : « Non vuole essere capita ${ }^{33}$ ». Il s'agit d'une mère qui demeure, fière, dans sa posture de femme tournée, de femme sans visage, sans compassion, qui refuse tout rapprochement : mental, corporel. Quand elle choisit d'observer, elle le fait à travers une perspective manquante. Ses jumelles sont renversées : elles déforment les objets, les choses. Elles parviennent à fausser le profil de ses mêmes enfants : « La madre li guarda / col binocolo capovolto, / li allontana / i bambini opachi ${ }^{34} »$. Eux, les enfants de la nuit, ne sont rien d'autre, pour elle, que des entités insondables, privées d'identité.

Le constat de Candiani est implacable. Les efforts sont vains : rien ne change, rien ne peut changer. La mère reste enfermée dans sa cage de folie, d'absence et de silences. Malgré les tentatives de communication, aux yeux d' « Io » elle continue « implacabile a non veder[la $]^{35} »$.

\footnotetext{
${ }^{30}$ C. LiVia CANDIANI, « Vista dalla luna », op. cit., p . 20.

${ }^{31}$ Ibid., p. 25.

${ }^{32}$ Ibid., p. 31.

${ }^{33}$ Ivi.

${ }^{34}$ Ibid., p. 34.

${ }^{35}$ Ibid., p. 42.
} 
Les conséquences de ces comportements réitérés produisent une forme de conscience malheureuse chez le sujet. « Io » sait bien qu'elle n'a pas été désirée. Elle est venue au monde, mais elle a été, tout de suite, « consegnata / al buio della notte ${ }^{36}$ ». Le Je poétique se perçoit, dans sa conscience lucide, comme un être à part, qui ne peut pas accéder à l'horizon de sa génitrice. « Io » se figure comme un « numero sfuggito all'ultima / delle [s]ue somme, la cifra / che non torna ${ }^{37} \gg$. Elle est un accident; un numéro effacé à l'intérieur d'une arithmétique affective qui ne prévoit pas sa présence. Cette mère-aveugle est, finalement, comparable à l'assassin du poème La porta. Un individu qui habite la chambre d'à côté, mais qui demeure incapable d'empathie, d'émotion. Quelqu'un qui est insouciant de l'autre - « Incurante ${ }^{38} »-$ quelqu'un qui, prisonnier dans son monde impénétrable, ne fait que répéter, chaque jour, le même geste. Fermer une porte, empêcher toute sorte de communication, soustraire le regard : « Chiude / la porta. / Identica. / Vuota ${ }^{39} »$.

De son côté, le paradigme de la mère-cannibale montre comment, cette même absence, peut être porteuse d'un côté cruel, faisant référence au domaine du corps. La cécité conserve, dans ses caractéristiques, une forme de dénis qui peut se transformer un désir de suppression. «Io » imagine sa mère décapitée. Elle la voit dans une flaque de sang : « la testa della mamma / la mamma contro il muro / lascia strisce rosse ${ }^{40} »$. Dépossédée de son corps, la mère est exposée comme une idole païenne douée d'une anatomie de fantoche. Le corps de la mère est un corps massacré qui reproduit, dans sa morphologie de cadavre, les signes d'un manque à être qui devient, dans ce contexte : évident. Cette même mère est celle qui n'a pas de cœur, qui ne sait pas soigner, écouter. Sa sensibilité est bloquée par un portail qui interdit tout accès : «la mamma ha un cancello / nero e acuminato ferisce ${ }^{41} »$. C'est un barrage qui empêche toute possibilité d'accueil vers ceux que Candiani définit comme les enfants imprévus : «i bambini imprevisti ${ }^{42}$ ». «Io » est, justement, cette petite fille imprévue ; une enfant qui n'a pas été désirée, une enfant qui est susceptible d'être dévorée par cette mère-cannibale qui avale l'autre faute de pouvoir l'aimer. Le portail acéré - synonyme du cœur maternel - blesse les doigts de ces enfants. Ces mains tendues dans l'espoir de recevoir, ces mêmes mains en quête d'une parole d'amour, se heurtent contre une porte fermée, un cœur inaccessible.

\footnotetext{
${ }^{36}$ Ibid., p. 41.

${ }^{37}$ Ibid., p. 43.

${ }^{38}$ C. LiVIA CANDIANI, « La porta », op. cit., p. 125.

${ }^{39} \mathrm{Ivi}$.

${ }^{40}$ C. Livia CANDiAni, « Vista dalla luna », op. cit., p. 21.

${ }^{41}$ Ibid., p. 32.

${ }^{42}$ Ivi.
} 
La mère-cannibale est, donc, cette figure noire qui devient, dans certains poèmes de Vista dalla luna, un grand « Tu » contre lequel « Io » se bat dans un jeu d'accusations, de remarques lucides. Le Je poétique ne fait jamais marche arrière. En revanche il cherche, sans cesse, à signifier la portée des dégâts provoqués par le manque d'amour. L'enfant-je qui se lance contre cette mère-tu est une petite fille qui, en dépit de la présence de ses parents, saisit sa condition d'orphanité. Dans ce contexte, il est préférable de tomber dans le bras du croque-mitaine, de cet « uomo nero » qui, paradoxalement, semble être plus accueillant que la « gonna piovosa / a cui non si aggrappa senso $»^{43}$ de la mère. Les jupons maternels ne constituent pas un point d'accroche. Le sens échappe et la recherche d'une parole - d'un signe d'affection qui est toujours refusé - jette le sujet dans le précipice de l'absurdité. « Io » accuse le « Tu » maternel de ne l'avoir pas aimée, d'être passée comme une ombre : « il tuo feroce passare / intatta dalla vita, assente $e^{44} »$. La mère est ce lieu incapable de se faire abri - « tu non sei casa ${ }^{45} »$. Elle est cette chair qui met au monde, qui donne naissance, mais qui, finalement, préfère tourner le regard. En faisant ainsi, elle a livré sa propre fille aux abysses de la nuit : « quando mi hai nata consegnata / al buio della notte ${ }^{46} \gg$.

Qu'est-ce qu'il reste, alors, à ce sujet perdu ? La petite fille, démunie, déchirée dans son identité, ne possède rien d'autre que son expression. Un cumul de mots qu'elle-même a dû apprivoiser pour pouvoir accéder au monde, pour entrevoir une brèche contre la violence avalée tout au long de son enfance. Ce qui perdure, après la bombe, c'est ce cri muet. Un cri qui se lève et qui s'incarne, prophétiquement, dans la littérature et, notamment, dans la poésie. Cette dernière constitue la membrane transparente qui se forge entre la dicibilité du monde et l'absence de tout discours. Candiani le précise dans une interview où elle explique comment la poésie possède l'étrange, étonnante, capacité à « sauver la réalité ${ }^{47}$ ». Après la catastrophe du non-amour, c'est l'idiome qui survit, qui permet l'accroche, qui trace un parcours permettant de se relever. Le langage résiste et la parole, de son côté, est ce pont entre l'écriture et le désert. Dans la poésie se renouvelle le prodige de deux nécessités humaines : le besoin de silence, et le besoin d'expression. Les espaces entre les mots répondent à la nécessité - vitale - d'absences, de suspension. Et la parole, maîtresse suprême - moteur de significations - répond, dans son hétérogénéité, dans sa capacité nourrissante - maternelle - à ce besoin d'énonciation qui permet au sujet de se dire. D'être, finalement : écouté. Ce n'est que dans l'espace de la page que « Tu»

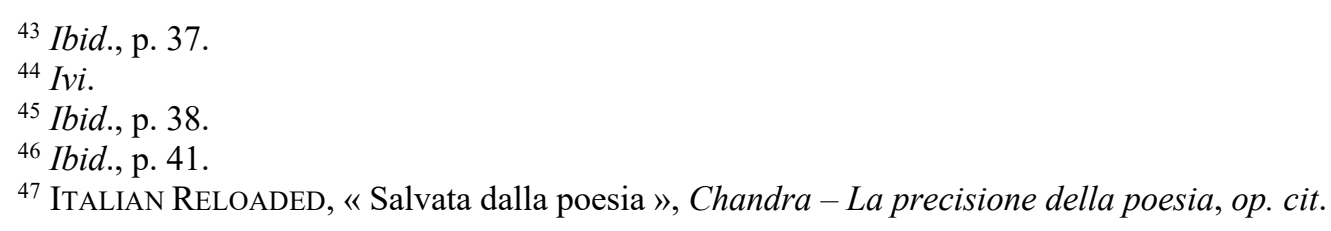


et «Io» peuvent finalement se réunir : « io e tu svaniscono / nell'identico urlo $^{48}$ ». Ils retrouvent, ainsi, dans l'écriture, un point de contact : une légère forme de réparation qui débouche, toutefois, vers un cri désespéré. Le dernier appel avant l'abîme.

\section{S'édifier après l'horreur : le choix de la parole}

Nous avons pu analyser comment la perspective de l'enfance - nous avons même parlé de posture - permet à la poétesse de pénétrer dans la folie d'une éducation au négatif. Savoir habiter cette saison de l'existence est une manière, pour Candiani, d'adopter l'exactitude de la parole. Dans sa préface au volume, elle précise sa position au sujet de l'expression poétique : « Non sono un cantore di astrattezze ${ }^{49}$ ». La poésie est, donc, ce lieu où la parole assume un périmètre précis. C'est une parole qui, nous l'avons vu, se fait plan - « mappa » - à travers laquelle pouvoir lire le monde, pouvoir trouver sa propre place.

La poétesse semble reproduire, dans ce contexte, la leçon d'I. Calvino, réitérée à plusieurs reprises dans son essai Le lezioni americane ${ }^{50}$. Car, pour contraster la « perte de la forme ${ }^{51} »$, la désintégration du sujet et, par conséquent, l'anéantissement de toute possibilité argumentative, il faut proposer - dresser - une idée de littérature. Le potentiel du langage poétique permet, en effet, de contraster les dérives d'un quotidien qui sombre vers la folie, le non-sens, l'absence de compréhension. La parole est cette arme que Candiani utilise en connaissance de cause. Il s'agit d'un outil qui développe une tension interne vers un désir d' « exactitude $»^{52}$ qui semble, dans son potentiel de précision, fournir une clé de lecture pour signifier la douleur, pour la faire ressurgir dans la page.

Cette idée est renforcée par le besoin - la nécessité - de nommer les objets. Le seul contact avec le monde des vivants passe, en effet, par la capacité à savoir reconnaître les éléments du quotidien. Une table, une chaise, une fourchette : «mentre i grandi si sbranano / tavolo sedia forchetta ${ }^{53} »$. C'est ainsi qu' « Io », la petite fille venue de la lune - cette enfant qui n'est pas de ce monde, qui ne trouve pas d'accueil auprès de sa famille - utilise les objets, les arbres, la nature, pour chercher à structurer sa personnalité. Face aux blessures, face à la souffrance

\footnotetext{
${ }^{48}$ C. LiVIa CANDIANI, « Vista dalla luna », op. cit., p. 48.

${ }^{49}$ C. LIVIA CANDIANI, « Essere visti dalla luna », Vista dalla luna, op. cit., p. 14.

${ }^{50}$ I. CALVInO, Le lezioni americane [1993], Milano, Mondadori, 2016.

${ }^{51}$ Ibid., p. 61.

52 Ibid., p. 67.

${ }^{53}$ C. Livia CANDIANI, « Vista dalla luna », op. cit., p. 18.
} 
physique, aux coups reçus sans raison, «Io » s'accroche à la nature, au mystère des fleurs : « non ho paura / di nessuno Io, perché i calci / fioriscono le viole le viole ${ }^{54}$ ».

Résister à la violence signifie garder, dans sa tête, une perspective lucide : celle qui permet au sujet de comprendre que l'existence n'est pas un magma figé, immuable. L'univers n'est pas celui de la folie, celui indiqué par les parents : les ténèbres, l'horreur. L'univers est un arbre, une fleur. Il s'incarne dans la tête d'une jeune fille qui choisit la parole, qui invente un langage autre pour signifier son monde d'exilée. Pour Candiani, il s'agit d'une jeune fille douée d'un esprit qui sait fleurir. Contre les menaces parentales : « una testa fiorisce / [...] la testa di Io $^{55}$ ». L'enfance est, alors, un territoire lumineux qui s'oppose à l'obscurité. L'enfance, saison massacrée par l'incompréhension, devient une possibilité d'ouverture vers un monde édénique, intouchable, ouvert à une forme d'émerveillement que les adultes ignorent. Candiani semble s'appuyer sur l'enseignement d'E. Montale qui, dans son poème Riviere, alterne ce jeu d'obscurité et de splendeur en faisant référence à sa terre d'origine : à son enfance en Ligurie. Revenir en arrière signifie accepter la tromperie de la mémoire, accepter de revivre l'enfance pour ce qu'elle représente : un tournant traversé de « ricordi lieti-e atroci ${ }^{56}$ ». Mais cette même enfance montalienne, consommée entre les après-midi ensoleillés et l'ombre des inquiétudes intimes, est la même qui cherche, grâce au choix de la poésie, une manière pour signifier l'importance d'un langage qui sache exprimer la complexité des individus, la fragilité de la mémoire. Le Je lyrique s'anéantit dans la vision. Il se dissipe dans la contemplation et il ne retrouve une forme de consistance que dans une langue capable de traduire sa subjectivité. La parole poétique est cet appendice du corps, le complément de la pensée. Elle représente l'unique moyen pour condenser la fièvre des désirs et le tourment des peines. Refleurir est, pour E. Montale et Candiani, une manière d'adhérer à la nature, ultime dépositaire de la vérité d'un sujet partagé entre l'obscurité et la lumière : « un urger folle / di voci verso un esito ; e nel sole / che v'investe, riviere, / rifiorire $!^{57}$ »).

En empruntant la voie d'une nouvelle - deuxième - naissance à travers la parole, Candiani continue dans son parallèle avec la nature. Les branches hivernales apparaissent, dans leur nudité, comme l'emblème d'un espoir silencieux, d'une promesse gentille : celle du printemps qui se prépare. L'arbre dépouillé, photographié dans son immuabilité, garde en lui la volonté de fleurir à nouveau. Il ne craint pas la neige : il lutte, seul, contre le froid, la solitude, l'angoisse

\footnotetext{
${ }^{54}$ Ibid., p. 22.

${ }^{55}$ Ibid., p. 23.

${ }^{56}$ E. Montale, « Riviere », Tutte le poesie, Milano, Mondadori, 2006, p. 104.

${ }^{57}$ Ibid., p. 104.
} 
d'une longue nuit qui semble infinie. Le poète, pour Candiani, est celui qui n'oublie jamais la leçon de la nature. Celui qui s'accroche, justement, à la branche solitaire d'un arbre abandonné en la percevant comme une sœur, comme une semblable. C'est la capacité à générer, malgré tout, une nouvelle vie : « i bambini opachi / [...] legati ai rami / fioriscono per necessità ${ }^{58}$ ». Cette fleuraison thérapeutique, susceptible de donner une nouvelle sève au sujet fracturé, se traduit, parallèlement, dans une sorte de prière laïque. Candiani semble, une fois encore, invoquer les vers d'E. Montale qui, dans son Proteggetemi ${ }^{59}$, invoque la protection des certains gardiens invisibles. Il faut trouver des esprits capables de préserver les poètes contre les horreurs du quotidien - l'oubli - contre les absences qui fracturent, le mensonge du succès, la soif de reconnaissance. Être protégé, pour E. Montale et pour Candiani, ne signifie rien d'autre que chercher une échappatoire à travers cette même parole poétique qui peut se révéler trompeuse, caduque. Pour la poétesse, il s'agit d'une parole précaire, poreuse, qui se concrétise dans la figure d'un ange. L'espoir s'incarne, justement, dans cet être insaisissable - invisible à la multitude des sujets - capable, toutefois, de réparer les blessures du cœur. L'ange est l'attente qui se fait discours, langue, expression : «Cucimi Angelo un largo petto / per gli anni che non contano $^{60} »$. Mais être protégé, se protéger soi-même, signifie aussi savoir trouver un lieu mental, physique - où pouvoir dormir : « un ripostiglio di sogni caldi ${ }^{61}$ ». Une région où pouvoir se défendre contre les cris des malades, la folie parentale, la précarité des rapports. Dormir est un droit inaliénable ${ }^{62}$ que Candiani revendique comme le fondement de toute construction de soi. Car, pour elle, se reposer équivaut à se sentir à l'abri de toute violence : « Casa è ovunque io senta tana. E cioè un posto dove le persone hanno fatto una scelta di non violenza $^{63} »$. La maison devrait être un lieu où les gens parviennent à communiquer, à échanger dans le respect de l'autre.

Affronter les ténèbres signifie, dans la poésie de Candiani, trouver la force de les observer droit dans les yeux. «Io», dans sa recherche d'identification, ne se soustrait jamais à l'apprentissage de la parole. Il s'agit d'un personnage qui choisit de raconter l'authenticité du mal sans jamais baisser le regard : « Io / ma quando salta / nel buio vuoto, / lo fa a occhi aperti / e a piedi pari per tuffarlo / tutto intero / il male ${ }^{64}$ ». L'image de la Gorgone revient en filigrane en annonçant non seulement la posture morale de la poétesse, mais surtout son parcours à

\footnotetext{
${ }^{58}$ C. LiVia CANDiAnI, « Vista dalla luna », op. cit., p. 34.

${ }^{59}$ E. Montale, « Proteggetemi », Tutte le poesie, op. cit., p. 628.

${ }^{60}$ C. Livia CANDIANI, « Vista dalla luna », op. cit., p. 26.

${ }^{61}$ Ibid., p. 24.

${ }^{62}$ ItAlian ReloAded, « Casa », Chandra - La precisione della poesia, op. cit.

${ }^{63}$ Ivi.

${ }^{64}$ C. Livia CANDiAnI, « Vista dalla luna », op. cit., p. 47.
} 
travers la poésie. La littérature, en reprenant l'enseignement d'I. Calvino concernant la Légèreté, est ce bouclier argenté capable de permettre aux poètes de fixer le mal, de l'habiter par une voie autre. Il faut opposer aux regards qui tuent, l'intelligence d'un instrument qui reflet la vérité. C'est l'enseignement de Persée qui parvient à voir Méduse sans mourir. La poésie est, donc, ce même bouclier ; un filtre qui se fait support d'une série de narrations, mais qui choisit, dans l'usage d'une parole-tiroir - une parole d'abondances et de privation - de raconter le monde à travers une perspective oblique. Autre.

La parole poétique de Candiani - celle qui l'a sauvé du précipice, celle qui lui a fourni une accroche indispensable pour remonter la pente de son désespoir - traduit, donc, cette vision parallèle. C'est une perspective critique, et donc poétique, qui se nourrit de l'exactitude calvinienne, qui embrasse l'idée d'une légèreté qui n'est jamais superficialité, mais plutôt poids d'une connaissance qui se fait, soudainement, subtile : aérienne.

Car, c'est à travers cette langue particulière - une langue qu'il faut savoir lire, écouter, protéger - qui passe la compréhension du monde et l'appropriation de celle que P. P. Pasolini indiquait comme une idée de beauté6 ${ }^{5}$ : l'intelligence, la singularité de chaque individu. Cet idiome qui vient de l'enfance - et qui se sert de l'enfance pour lire le monde - est « un fuoco d'alfabeto / senza lingua senza / senso $^{66}$ ». Il s'agit d'un langage indéchiffrable qui brûle d'angoisse, qui s'ouvre à l'incompréhension du non-amour, mais qui garde, dans sa cacophonie expressive, la consistance de la grâce.

C'est ainsi que ce besoin qui se fait néant, qui embrasse l'annihilation du sujet - « sconfinato è solo il niente ${ }^{67} \gg$ - ouvre la voie à une perspective critique que les lecteurs - tous - doivent pouvoir adopter. Il faut savoir accueillir la parole poétique comme une voix nouvelle, qui n'a pas de patrie. C'est une parole capable de nourrir et, paradoxalement, une parole de la soustraction, qui descend dans les profondeurs de la souffrance dans l'objectif de pouvoir la comprendre, l'apprivoiser. De la restituer. Cette parole, nous répète Candiani, est celle qui se fait déchirure - du sujet, de la page - et qui devient, paradoxalement, une fissure à travers laquelle faire passer la lumière. En italien, Chandra joue sur l'assonance entre « ferita »- la blessure - et la $«$ feritoia $»^{68}$, cette fente qui nous permet d'observer l'ennemi tout en nous

\footnotetext{
${ }^{65}$ P. P. PAsolinI, « Siamo belli, dunque deturpiamoci », Lettere Luterane. Il progresso come falso progresso, introduzione di Alfonso Berardinelli, Torino, Einaudi, 2003.

${ }^{66}$ C. LIVIA CANDIANI, «Vista dalla luna », op. cit., p. 27.

${ }^{67}$ Ibid., p. 30.

${ }^{68}$ M. FERRETTI, «Le ferite », Psychiatry on line Italia video channel, 28 novembre 2017.
} 
laissant envahir par la lumière qui pénètre à travers les ouvertures : «Questo silenzio, non un altro / attimo, ma la fragorosa apertura ${ }^{69} »$.

Contre l'obscurité, la parole est ce même bouclier qui s'ouvre sur le néant afin de pouvoir le restituer. Elle est une plume qui se fait sujet, qui se fait $J e-«$ Io »- voix capable de transporter l'individu brisé vers l'autre rive, celle de l'énonciation, de la connaissance : « Io è la penna ${ }^{70}$ ». La parole est poudre à canon - « Le parole, / d'amore, / si frantumano / fino a farsi / polvere. / Da sparo $^{71} »-$ permettant au sujet de se défendre à travers l'arme de l'expression. On se dit dans les vers. On existe grâce à la possibilité de nommer le monde. Ce n'est qu'à travers ces instruments qu'il est possible d'accéder à la complexité de l'existence. Une existence qui est faite de fractures - le blanc parmi les mots, dirait Candiani - et qui est traversée de paroles solitaires qui percent l'obscurité du vocabulaire. Une existence, in fine, qui grâce à la poésie permet à cette petite fille venue de la lune - cette enfant-boxeuse, en souvenir du recueil $L a$ bambina pugile - de s'habiller de monde, de se faire elle-même « mappe », plan, et donc destin.

Amo il bianco tra le parole

$[\ldots]$

amo la parola che spunta

solitaria

sullo specchio buio del vocabolario

[...]

Quando veste il mondo, quando lo rivela, quando fa mappa, quando fa destino. ${ }^{72}$

\footnotetext{
${ }^{69}$ C. Livia CANDiAni, « Vista dalla luna », op. cit., p. 49.

${ }^{70}$ Ibid., p. 43.

${ }^{71}$ C. LIVIA CANDIANI, « La porta », op. cit., p. 77.

${ }^{72}$ C. Livia CANDIANI, La bambina pugile, op. cit., p. 31.
} 